BNL- 73999-2005-CP

Dynamic Aperture Optimization for Low Emittance Light Sources

S. Kramer and J. Bengtsson

NSLS, Brookhaven National Laboratory, Upton, NY

May, 2005

\title{
National Synchrotron Light Source
}

Brookhaven National Laboratory

Managed by

Brookhaven Science Associates

Upton, NY 11973

Under Contract with the United States Department of Energy

Contract Number DE-AC02-98CH10886 


\section{DISCLAIMER}

This report was prepared as an account of work sponsored by an agency of the United States Government. Neither the United States Government nor any agency thereof, nor any of their employees, nor any of their contractors, subcontractors or their employees, makes any warranty, express or implied, or assumes any legal liability or responsibility for the accuracy, completeness, or any third party's use or the results of such use of any information, apparatus, product, or process disclosed, or represents that its use would not infringe privately owned rights. Reference herein to any specific commercial product, process, or service by trade name, trademark, manufacturer, or otherwise, does not necessary constitute or imply its endorsement, recommendation, or favoring by the United States Government or any agency thereof or its contractors or subcontractors. The views and opinions of authors expresses herein do not necessarily state to reflect those of the United States Government or any agency thereof. 


\title{
DYNAMIC APERTURE OPTIMIZATION FOR LOW EMITTANCE LIGHT SOURCES*
}

\author{
S.L. Kramer ${ }^{\#}$ and J. Bengtsson \\ BNL/NSLS, Upton, NY 11973, U.S.A.
}

\begin{abstract}
State of the art low emittance light source lattices, require small bend angle dipole magnets and strong quadrupoles. This in turn creates large chromaticity and small value of dispersion in the lattice. To counter the high linear chromaticity, strong sextupoles are required which limit the dynamic aperture. Traditional methods for expanding the dynamic aperture use harmonic sextupoles to counter the tune shift with amplitude. This has been successful up to now, but is non-deterministic and limited as the sextupole strength increases, driving higher order nonlinearities. We have taken a different approach that makes use of the tune flexibility of a TBA lattice to minimize the lowest order nonlinearities, freeing the harmonic sextupoles to counter the higher order nonlinearities. This procedure is being used to improve the nonlinear dynamics of the NSLS-II lattice.
\end{abstract}

\section{INTRODUCTION}

The National Synchrotron Light Source (NSLS) was one of the first $2^{\text {nd }}$ generation light sources and has operated more than 20 years, with a large user community. The existing storage rings, have been improved over the years, but the demand for more undulator beam lines with higher brilliance, can not be accommodated within the present facility. Therefore a proposal to upgrade the facility with a $3^{\text {rd }}$ generation, ultra-high brilliance storage ring has been presented (NSLS-II). The energy of the storage ring is $3 \mathrm{GeV}$, which is sufficient for producing undulator radiation beams in the $0.3-20 \mathrm{KeV}$ range based on the new in vacuum micro-gap undulator technology. The storage ring requirements to meet the users goals are:

Ultra-low horizontal emittance $<1.5 \mathrm{~nm}$ (achromatic),

Diffraction limited vertical emittance at $12 \mathrm{KeV}$,

Stored currents to $500 \mathrm{~mA}$,

At least 20 ID straight section for $>5 \mathrm{~m}$ undulators,

Top-off injection, and

Future upgrade potential to an ERL.

The lattice structures being studied for NSLS-II are Three Bend Achromatic (TBA) and Double Bend Achromatic (DBA) lattices. The one that meets all requirements is the TBA lattice and will be discussed here.

\section{LINEAR LATTICE DESIGN}

The TBA lattice composed of iso-magnet dipoles and with $\theta_{p}$ total bend angle per period, could have a minimum emittance [1] given by

\# skramer@bnl.gov

* Work supported by U.S. DOE, Contract No.DE-AC02-98CH10886

$$
\varepsilon_{\text {METBA }}=\frac{C_{q} \gamma^{2}}{4 \sqrt{15}} \frac{\theta_{p}^{3}}{J_{x}(3.44)^{3}}
$$

where $\gamma$ is the relativistic energy, $J_{x}$ is the horizontal partition factor and $C_{q}=3.84 * 10^{-13} \mathrm{~m}$. At $3 \mathrm{GeV}$ a 24 period ring has $\varepsilon_{\text {METBA }} \approx 0.38 \mathrm{~nm}$ or a factor of more than 4 times less than the desired emittance, a comfortable factor in line with that achieved at existing light sources. The DBA lattice, having a factor of 5 more emittance per period [1], would require 32 or more period lattice to achieve the emittance goal.

Achieving even close to the minimum emittance in a small circumference ring requires large betatron phase advance and strong quadrupoles. Correcting for the resulting high chromaticity requires strong sextupoles reducing the dynamic apertures (DA) necessary for injection and good lifetime. The basic DBA lattice is very restrictive since low emittance requires the horizontal tune to be close to unity per period. Adding quadrupole families can provide the greater phase advance between the dipoles necessary to approach the minimum emittance condition, but causes the chromaticity to increase rapidly. The TBA lattice having two different dipoles, allows the phase advance in the dispersion region to be varied over a greater range, while maintaining the low emittance condition. Consequently we have found the TBA lattice more flexible in tuning the betatron phase advance per period, while maintaining the emittance goal. This flexibility will be utilized for improving the ability to control the DA discussed below.

To achieve $\varepsilon_{\text {МЕTвA }}$ requires a ratio of the outer to inner bend angles of $\theta_{o}=\sqrt[3]{3} \theta_{i}$ [1]. $\varepsilon_{\text {METBA }}$ also requires a large phase advance between the dipoles yielding a large chromaticity. Since $\varepsilon_{\text {METBA }}$ is never achieved in practice, we have chosen a simpler ratio of the dipole bend angles of $\theta_{o}=2 \theta_{i}$. This choice has a similar value of the minimum emittance, which also cannot be achieved. This bend angle ratio is similar to the choice of $\theta_{o}=1.75 \theta_{i}$ in the SLS TBA lattice [2]. We have also reduced the number of dispersion quadrupoles from four to three families, by using gradient dipoles. This provides for the vertical focusing but also increases $\mathrm{Jx}$, thereby reducing $\varepsilon_{x}$. This reduction of quadrupoles allowed space for a $4^{\text {th }}$ family of chromatic sextupoles that will help tune chromatic corrections to the DA. The basic TBA 24 period lattice functions are shown in Figure (1). 
The greatest advantage of the TBA lattice is that the emittance can be maintained to a constant value while the cell tune is varied over a large range. This is shown in Figure (2) where the cell tune is varied while holding the emittance fixed to $1.46 \mathrm{~nm}$.

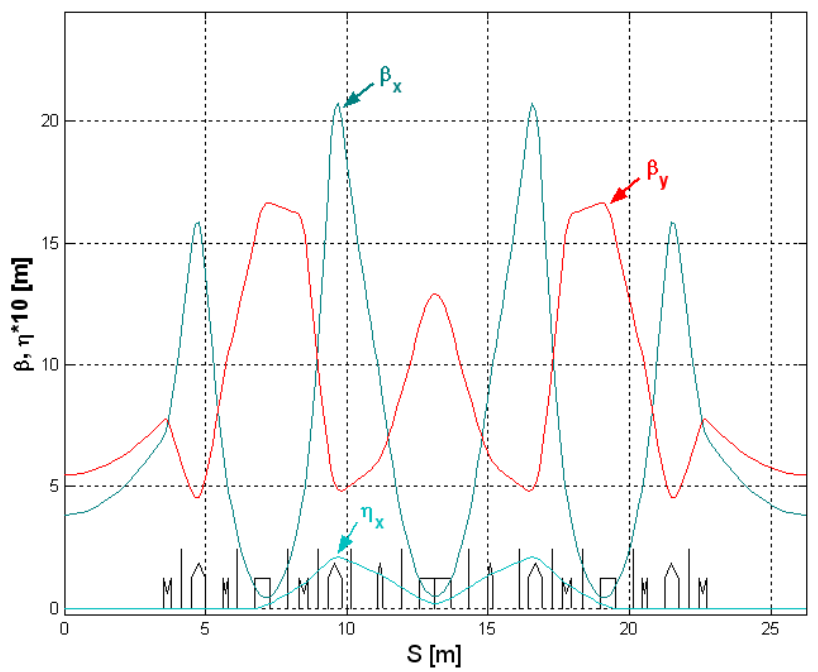

Figure (1) Lattice functions for 24 period lattice with $\varepsilon_{x}=1.46 n m,\left(v_{x}, v_{y}\right)=(1.562,0.560) /$ cell.

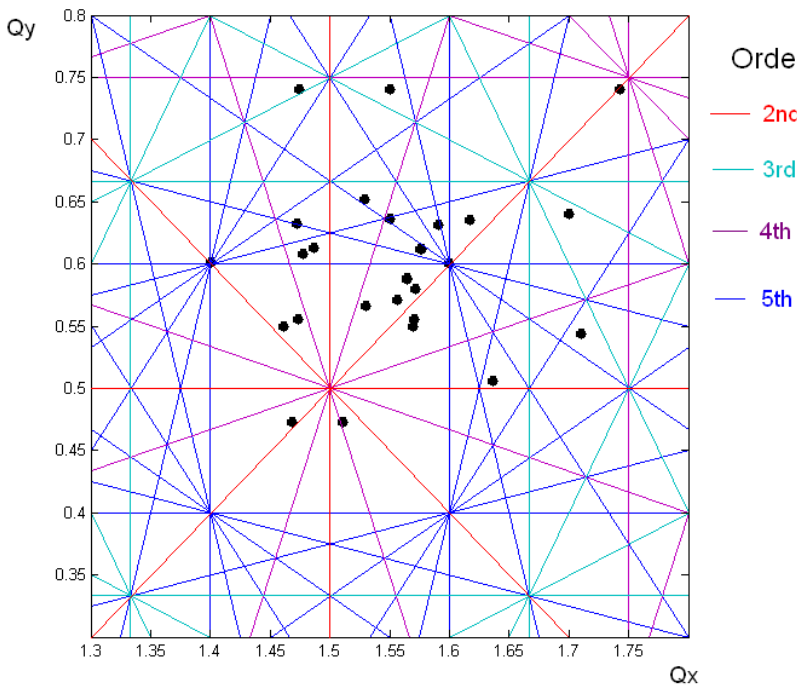

Figure (2) Working point diagram, showing cell tune flexibility for the TBA lattice, with emittance of $1.46 \mathrm{~nm}$.

\section{DYNAMIC APERTURE OPTIMIZATION}

Most light sources have had to deal with the reduced DA from the strong sextupoles necessary for tuning linear chromaticity. Early efforts have added harmonic sextupoles to reduce effects of leading order systematic resonances and tune shifts with amplitude introduced by the chromatic sextupoles. This approach was based on harmonic analysis of the Hamiltonian using $1^{\text {st }}$ and $2^{\text {nd }}$ order perturbation theory for the slowly varying terms [3]. As the push for lower emittance drove sextupoles even stronger, the shortcomings of the resonance approach have become apparent.
Alternatively, a more comprehensive approach has based on the Lie series representation of the one-turn map, with the generator a power series in the multipole strength [4]. This approach provided a method for controlling the non-linear lattice properties for the SLS lattice, which yielded a robust DA sufficient for injection and operation [5]. The requirements for NSLS-II are pushing the non-linear properties of the lattice to a level where this is no longer sufficient. While the DA with 4chromatic and 3-geometric sextupole families optimized with this procedure $[4,6]$, gave adequate results for an ideal lattice, it proved insufficient when engineering tolerances were included. This can be seen from the large non-linear tune shift with amplitude, $J$, shown in Figure (3), which will excite non-structure resonances. To address this, the analytical method has been extended [7].

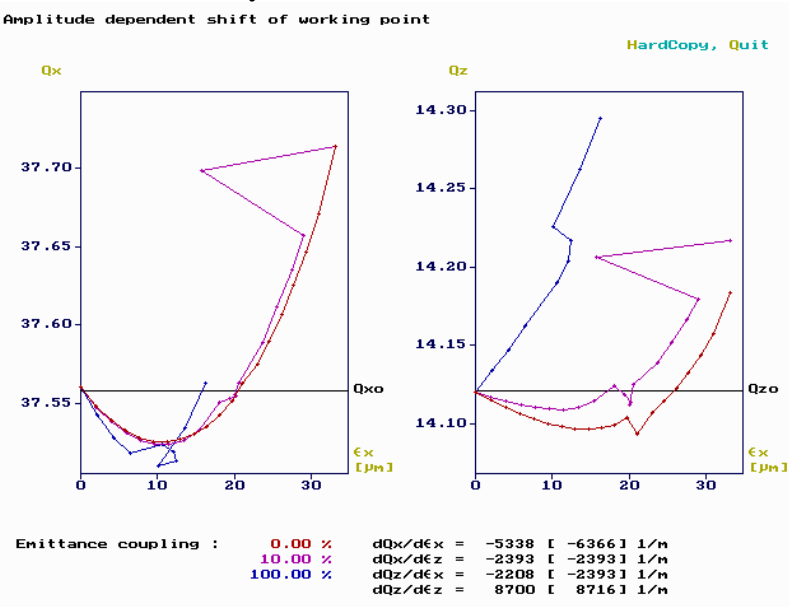

Figure (3) The betatron tunes Qx (left) and Qy (right) vs amplitude, $\varepsilon_{x}=2 \mathrm{~J}$, for 3 coupling ratios $(0,10$, $100 \%)$ for the basic 24 period lattice with a $2^{\text {nd }}$ order optimization using 7 sextupole families.

\section{WORKING POINT SEARCH WITH HIGHER ORDER OPTIMIZATION}

The one-turn map $\mathcal{M}$, can be expressed as [8]:

$$
\mathcal{M}=\mathcal{A}^{-1}\left[e^{: h:}(R \circ \Delta R)\right] \mathcal{A}
$$

where $: h:=: h_{3}+h_{4}+\ldots h_{8}$ : the generator expansion for the nonlinear fields, $\mathbb{R}$ is the linear tune operator with $\Delta R$ a small tune shift operator and $\mathcal{A}$ is a linear canonical transformation to normalized phase space. Previous attempts tried to minimize the driving terms up $\mathrm{h}_{4}$ or $2^{\text {nd }}$ order in the multipole strength $\left(\mathrm{b}_{\mathrm{n}}\right)$ was done by adding geometric sextupoles with appropriate phase and strength in the lattice [9]. This attempt to optimize the DA used a cell tune close to $v_{x, y} \approx \frac{7}{4}, \frac{3}{4}$ to cancel the $2^{\text {nd }}$ order sextupole driving terms over 4 cells. This works in principle for non-interleaved sextupoles but isn't a clear advantage once strong interleaved sextupoles are introduced. 
Our tune shift with $\mathrm{J}$ shows dependence up to cubic terms in the J. Minimizing these terms will require expansion of the sextupole terms up to $h_{8}$, or $6^{\text {th }}$ order in $b_{n}$. In particular, the expansion used here, requires minimization of 52 terms:

- 27 geometric resonance modes,

- 12 amplitude dependent tune shift terms, and

- 13 chromatic terms.

With such a large number of terms, we have taken the approach of making the phase advance variable in $\Delta R$ to improve the cancellation over $\mathrm{N}$ cells. As a first attempt, we searched near a cell tune of $(1.6,0.6)$ for the basic 24 period lattice in Figure (1). This will provide leading order cancellation of the sextupole terms over 5 cells. Once the lattice functions are chosen, the driving terms are minimized up to $h_{8}$ using the 9 sextupole strengths, by the method described in Ref. [7] and then the DA for this solution is calculated by tracking with TRACY [10]. This procedure was iterated by scanning the cell tune, $\Delta v_{x, y} \rightarrow \Delta R$, over a small range. One such scan is shown in Figure (4) for the lattice in Figure (1). The tune values for several DA peaks are clearly evident, resulting from the cancellation of the terms in :h:, but the broadest peak is shown around the tunes in Figure (1). Since the chromatic terms are also part of the minimization, the DA is also maximized for the momentum aperture, as shown in Figure (5).

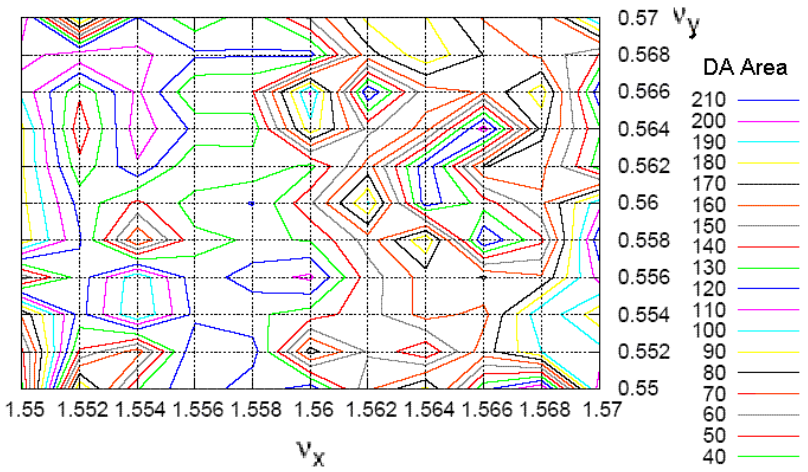

Figure (4) Working point scan ( $\Delta v_{x, y} \rightarrow \Delta R$ ) vs DA area, with :h: optimization performed at each point.

Additional study is required in order to demonstrate that this DA enhancement is maintained with real lattice errors included. Initial results have indicated the DA is robust for realistic errors, achievable with state of the art beam based alignment.

We have attempted to provide the tune flexibility using an additional cell with a variable cell tune. Figure (6) show one super period with 5 basic cells plus a large $\beta_{x}$ straight section cell for injection, to be used for optimizing the global tunes. This symmetry breaking of the lattice introduces 9 additional sextupole strengths, which can be accommodated in the optimization procedure. This has yielded a significant enhancement of the DA area. Work will continue on the working point optimization along with improved beta function matching for the undulator beam lines, however this method has already shown to be an effective way of dealing with the severe nonlinear distortions of this ultra low emittance lattice.

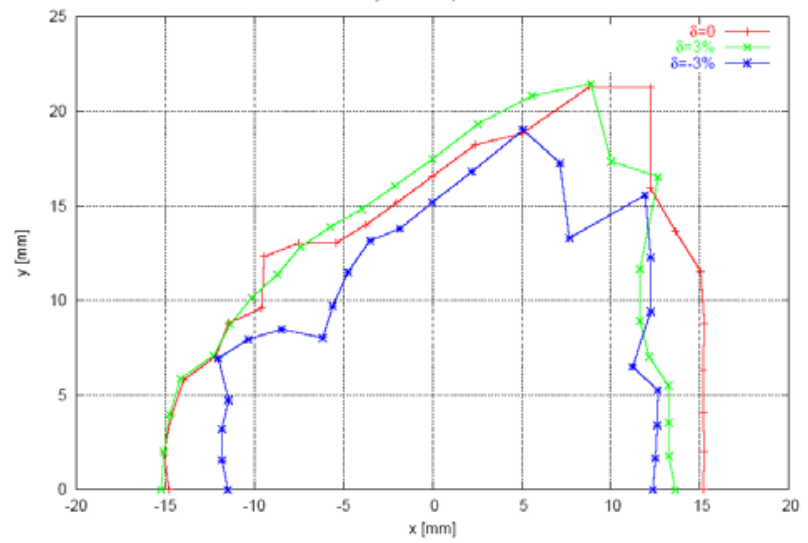

Figure (5) Optimized DA in ID ( $\left.\beta_{x}=3.9, \beta_{y}=5.5 \mathrm{~m}\right)$ with $\mathrm{dp} / \mathrm{p}=0,+/-3 \%$, (without synchrotron oscillations).

\section{REFERENCES}

[1] S.Y.Lee, Phys. Rev. E 54 (1996) p. 1940.

[2] SLS Design Handbook, (1998).

[3] M. Donald, PEP Note-311 (1979). E. Crosbie, PAC'87, p. 443 (1987).

[4] J. Bengtsson, SLS Note 9/97, (1997).

[5] A. Streun et al PAC01, p. 224 (2001).

[6] A. Streun, OPA manual and program, http://slsbd.psi.ch/ streun/opa/opa.html

[7] J. Bengtsson, these proceedings (2005).

[8] E. Forest, J. Math. Phys. 31 (1990) p. 1133.

[9] J. Bengtsson, et. al., NIM A404(1998) p.237.

[10] J. Bengtsson, TRACY-2 User Manual, unpublished.

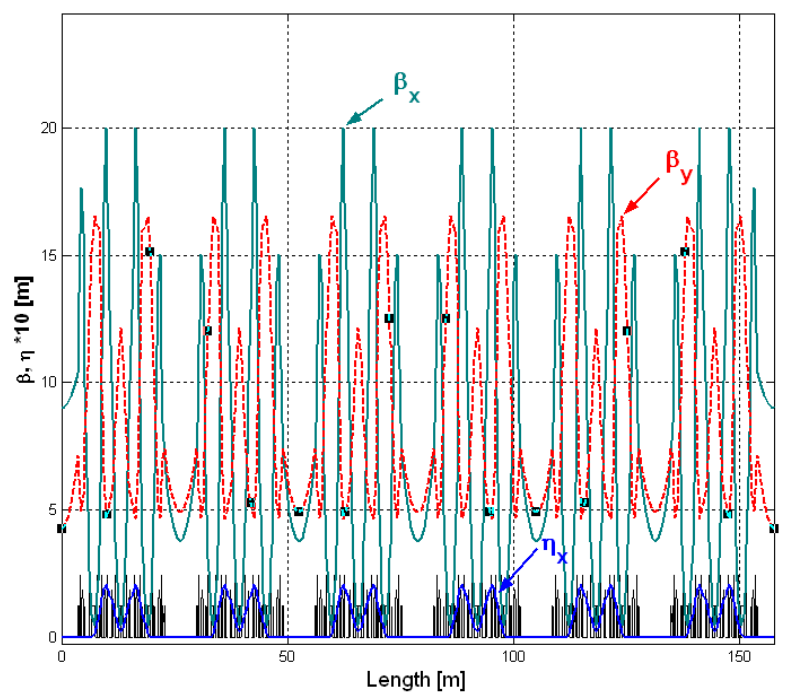

Figure (6) Lattice functions for a super period with 5 basic cells plus one high horizontal beta cell. 